Journal of

Synchrotron

Radiation

ISSN 1600-5775

Received 20 June 2014

Accepted 2 October 2014

\section{Functionalized synchrotron in-line phase-contrast computed tomography: a novel approach for simultaneous quantification of structural alterations and localization of barium-labelled alveolar macrophages within mouse lung samples}

\author{
Christian Dullin, ${ }^{\mathrm{a} *} \neq$ Simeone dal Monego, ${ }^{\mathrm{b}} \neq$ Emanuel Larsson,,${ }^{\mathrm{c}, \mathrm{d}, \mathrm{e}_{\ddagger}}$ \\ Sara Mohammadi, ${ }^{c}$ Martin Krenkel, ${ }^{f}$ Chiara Garrovo, ${ }^{\text {g }}$ Stefania Biffi, ${ }^{\text {g }}$ \\ Andrea Lorenzon, ${ }^{b}$ Andrea Markus, ${ }^{\text {h Joanna Napp, }}{ }^{\text {h,i,a }}$ Tim Salditt, ${ }^{f}$ \\ Agostino Accardo, ${ }^{d}$ Frauke Alves ${ }^{h, i, a}$ and Giuliana Tromba ${ }^{c_{9}}$ \\ anstitute for Diagnostic and Interventional Radiology, University Medical Center Göttingen, \\ Robert Koch Strasse 40, 37075 Göttingen, Germany, ${ }^{\mathbf{b}}$ Cluster in Biomedicine, AREA Science Park \\ Basovizza, Trieste, Italy, ${ }^{\mathbf{C}}$ Elettra Sincrotrone Trieste, Strada Statale 14, km 163.5 in AREA Science \\ Park, 34149 Basovizza (Trieste), Italy, ${ }^{\mathbf{d}}$ Department of Architecture and Engineering, University of \\ Trieste, Trieste, Italy, ${ }^{\mathbf{e}}$ Department of Physics, Chemistry and Biology, Linköping University, SE-581 \\ 83 Linkoeping, Sweden, 'Institute for X-ray Physics, University of Göttingen, Göttingen, Germany, \\ ${ }^{\mathrm{g}}$ Institute for Maternal and Child Health, IRCCS Burlo Garofolo, Trieste, Italy, 'hepartment of \\ Haematology and Medical Oncology, University Medical Center Göttingen, Robert Koch Strasse \\ 40, 37075 Göttingen, Germany, and 'Molecular Biology of Neuronal Signals, Max Planck Institute \\ for Experimental Medicine, Hermann-Rein-Strasse 3, 37075 Göttingen, Germany. \\ *E-mail: christian.dullin@med.uni-goettingen.de
}

Functionalized computed tomography (CT) in combination with labelled cells is virtually non-existent due to the limited sensitivity of X-ray-absorption-based imaging, but would be highly desirable to realise cell tracking studies in entire organisms. In this study we applied in-line free propagation X-ray phasecontrast CT (XPCT) in an allergic asthma mouse model to assess structural changes as well as the biodistribution of barium-labelled macrophages in lung tissue. Alveolar macrophages that were barium-sulfate-loaded and fluorescentlabelled were instilled intratracheally into asthmatic and control mice. Mice were sacrificed after $24 \mathrm{~h}$, lungs were kept in situ, inflated with air and scanned utilizing XPCT at the SYRMEP beamline (Elettra Synchrotron Light Source, Italy). Single-distance phase retrieval was used to generate data sets with ten times greater contrast-to-noise ratio than absorption-based CT (in our setup), thus allowing to depict and quantify structural hallmarks of asthmatic lungs such as reduced air volume, obstruction of airways and increased soft-tissue content. Furthermore, we found a higher concentration as well as a specific accumulation of the barium-labelled macrophages in asthmatic lung tissue. It is believe that XPCT will be beneficial in preclinical asthma research for both the assessment of therapeutic response as well as the analysis of the role of the recruitment of macrophages to inflammatory sites.

Keywords: phase-contrast CT; single-distance phase retrieval; functional CT imaging.

\section{Introduction}

Lung imaging, especially of the mouse, remains extremely challenging due to the small size and high porosity nature of the organ, which creates various problems like scattering for $\mathrm{X}$-ray and optical imaging techniques, susceptibility artefacts

$\ddagger$ These authors contributed equally to this work.

- These authors contributed equally to this work. in magnetic resonance imaging (MRI) and shadowing of medical ultrasound waves at the rib cage. Together with preclinical airway disease models, novel imaging technologies are becoming increasingly important for monitoring disease progression and the efficacy of treatment within the lung. Recent studies have either focused on functional aspects, utilizing near-infrared fluorescence (NIRF) imaging (Napp et al., 2010; Markus et al., 2014) or on the depiction of morpho- 
logical alterations, such as airway wall thickening using ex vivo micro-CT (Cortez-Retamozo et al., 2008; Sera et al., 2007). For tracking of ex vivo labelled immune cells in vivo a wide range of imaging modalities, for example MRI (Ahrens \& Bulte, 2013) and optical imaging (Bousso \& Moreau, 2012), have been reported in recent years. Radionuclide labelling of cells is the oldest technique for tracking immune cells, especially in whole body distribution studies in humans (Thakur, 1977). The application of CT in combination with novel contrast agents for cell tracking has just begun to be explored and is hampered by the poor sensitivity of CT combined with the low toleration of high concentrations of contrast media loaded into cells (Cormode et al., 2014). In order to visualize the biodistribution of labelled cells following injection, as well as to assess morphological alterations in great detail during inflammation and airway remodelling within the lung, the combination of high spatial resolution with increased sensitivity is highly desirable. An imaging technique that meets these requirements is the here-used in-line free-propagation $\mathrm{X}$-ray phase contrast CT (XPCT).

Since the very first application of X-rays for medical purposes by Konrad Roentgen in 1895, the imaging principle for detecting tissue-dependent variations by X-ray absorption has remained unchanged. Especially in applications related to visualization of soft-tissue as in mammography or in lung imaging, these differences in X-ray absorption are very weak, resulting in poor contrast. This contrast could be raised by lowering the X-ray photon energy; however, this would also increase the radiation dose deposition in the samples. Additionally, an energy level high enough to achieve sufficient penetration of the sample needs to be maintained. Due to these factors the absorption contrast in radiographs is limited, especially in clinical practice. In addition to absorption, a phase shift of the incident X-ray wavefront occurs within the sample. Despite being about 100 times stronger in low-density materials (Takeda et al., 1995), this effect has basically not been exploited in clinical routine to date, due to the fact that at least partial coherent X-rays are required, which can only be generated with micro-focus X-ray tubes, or with higher intensities, at synchrotron light sources (Nugent, 2010). This limitation notwithstanding, X-ray phase-contrast imaging has great potential, as it combines strong edge enhancement in radiographs with the fact that the advantage of phase contrast over conventional absorption contrast improves with increasing photon energy. Therefore, medical phase-contrast $\mathrm{X}$-ray imaging could potentially be performed at higher energies than in the actual absorption-based regime, which would reduce the dose deposition within the patient, especially in soft-tissue applications like mammography. There are already some clinical mammography systems on the market demonstrating a gain in image contrast due to phase effects (Tanaka et al., 2005). Besides the here-used in-line freepropagation XPCT, there are other phase-sensitive techniques such as grating interferometry (Pfeiffer et al., 2006). Grating interferometry would allow phase-contrast imaging even when conventional X-ray tubes are used. Notwithstanding that the clinical application of this technique is at the moment hampered by technical problems such as the magnitude of the applied radiation dose, grating interferometry also showed very promising results in lung imaging as demonstrated by Schleede et al. (2012).

Since absorption-based CT imaging in its nature shows low sensitivity, functional imaging approaches similar to those used in SPECT, PET or optical imaging (Nahrendorf et al., 2008) are virtually nonexistent. The potential of phase-sensitive techniques for lung imaging has been explored since the late 1990s at some synchrotron beamlines (Yagi et al., 1999; Kitchen et al., 2004; Lewis et al., 2005; Hooper et al., 2007, 2009). However, the increased sensitivity of XPCT in less dense materials might be well suited to realise CT functional imaging addressing both soft-tissue alterations and the distribution of heavy-ion-based contrast agents such as barium sulfate. Contrary to classical absorption-based CT, in XPCT edge enhancement caused by phase effects at tissue interfaces is superimposed on the contrast caused by tissue-specific differences in X-ray absorption. These interactions of X-rays with matter are described by the complex refractive index $\tilde{n}=1-\delta+i \beta$, where $\delta$ determines the phase shift and $\beta$ the absorption. In order to analyse the phase shift separately, the $\delta$ part of the signal needs to be decoupled by a phase-retrieval $(\mathrm{PhR})$ algorithm. It has already been demonstrated that the application of $\mathrm{PhR}$ is highly beneficial in the analysis of biological samples (Keyriläinen et al., 2010; Zhang et al., 2011; Yong et al., 2009; Gureyev et al., 2013) showing an up to 200fold improvement in the contrast-to-noise ratio (Beltran et al., 2011). A large variety of $\mathrm{PhR}$ algorithms have been reported so far, that can be loosely divided into multi- and singledistance techniques (Nugent, 2007).

Commonly used multi-distance $\mathrm{PhR}$ approaches are either based on the fact that the absorption part of the detected radiograph is constant whereas the impact of the phase effects varies with different sample-to-detector distances, or require a priori knowledge of the refractive indexes within the sample (Cloetens et al., 1999a; Beltran et al., 2010). Imaging with several sample-to-detector distances is disadvantageous because it crucially depends on a perfect alignment of the different scans and it is affected by variations in the X-ray beam, which often occur at synchrotron light sources. In contrast to that, single-distance $\mathrm{PhR}$ algorithms minimize the scanning time and are therefore especially suitable for the analysis of unfixed biological samples such as in situ mouse lungs imaged in this study, but they can strictly only be applied on objects expressing a constant $\delta$-to- $\beta$ ratio (Paganin et al., 2002). However, the applicability of this type of algorithm for soft-tissue samples was shown by Wu et al. (2005). Moreover, single-distance PhR followed by standard filtered backprojection reconstruction (FBP) was applied to generate three-dimensional (3D) data sets of mouse lungs predominately presenting the distribution of the $\delta$ part of the refractive index (Mohammadi et al., 2014).

The aim of this study was to develop a functional XPCT imaging approach (fXPCT) by exploiting the capability of alveolar macrophages $(M \Phi)$ to migrate to inflammatory sites within the lung using an ovalbumin induced experimental 
allergic airways disease model (Markus et al., 2014). In order to visualize the biodistribution of cells following intratracheal application, $\mathrm{M} \Phi$ of the immortalized alveolar macrophage cell line MH-S (Mbawuike \& Herscowitz, 1989) were ex vivo labelled by adding a contrast agent suitable for X-ray-based imaging directly to the cell culture media. A barium sulfate suspension was used, that is commonly applied in the clinic to mark the gastrointestinal tract in CT (Golder et al., 1991). Thereby, macrophages were intracellularly labelled and used as a specific probe instead of an in situ labelling approach by systemic administration of X-ray contrast agents. $M \Phi$ can engulf large particles by phagocytosis and can therefore easily be loaded with contrast agents and drugs in vitro (Trivedi et al., 2006), converting them into potential carriers for both diagnostic and therapeutic agents. Since they migrate to inflammatory sites they have already been exploited for delivery of various anti-inflammatory compounds (Bang et al., 2011; Moreira \& Hogaboam, 2011; Yang et al., 2012). Moreover, MФ were recently identified as one of the main effector cells in asthma (Bang et al., 2011; Moreira \& Hogaboam, 2011; Yang et al., 2012). Mizue et al. (2005) showed that, in the absence of the macrophage migration inhibitory factor (MIF), asthma could not be induced in MIF-deficient mice, and Chen et al. (2010a) demonstrated in an asthma mouse model that airway remodelling was successfully inhibited by a MIF antagonist.

With the unique capabilities of XPCT in combination with phase retrieval and the use of $M \Phi$ loaded with barium sulfate particles, we were able to simultaneously depict and quantify structural features and to illustrate in 3D the different accumulation sites of labelled $\mathrm{M} \Phi$ within asthmatic and control lungs. We believe that, by providing new quantitative functional and anatomical parameters and by using bariumlabelled immune cells in cell trafficking studies, this novel fXPCT approach may help to preclinically investigate complex and multi-factorial processes of inflammatory diseases.

\section{Material and methods}

\subsection{Preparation and labelling of macrophages}

The immortalized mouse alveolar $\mathrm{M} \Phi$ cell line MH-S (purchased from American Type Culture Collection, ATCC, USA) was maintained in RPMI medium, supplemented with $10 \%$ FCS and $0.05 \mathrm{mM}$ 2-mercaptoethanol (Mbawuike \& Herscowitz, 1989) in a humidified atmosphere at $5 \% \mathrm{CO}_{2}$ and $310 \mathrm{~K}$. For CT imaging, cells were loaded with a barium sulfate suspension, the clinically used contrast agent Micropaque CT (Guerbet, France) by co-incubating $1 \times 10^{6} \mathrm{cells} \mathrm{ml}^{-1}$ for $24 \mathrm{~h}$ with $3.5 \mu \mathrm{l}$ Micropaque $\mathrm{CT} / \mathrm{ml}$ cell media $(175 \mu \mathrm{g}$ barium sulfate/ml media), followed by two washing steps with phosphate buffer (PBS). Subsequently, for stable fluorescent labelling of the cell membrane, the $\mathrm{M} \Phi$ were incubated for 30 min with $5 \mu \mathrm{ml}^{-1}$ of the lipophilic dialkylcarbocyanine dye Vibrant DiD (DiD; Molecular Probes, Eugene, OR, USA; excitation maximum: $644 \mathrm{~nm}$; emission maximum: $665 \mathrm{~nm}$ ), followed by two washing steps with PBS. To test the loading efficacy of barium sulfate particles into $M \Phi$, a vial containing
$1 \times 10^{5}$ barium sulfate $\mathrm{M} \Phi$ resuspended in $100 \mu \mathrm{l}$ PBS was scanned with a normal bench-top microCT (eXplore locus SP, GE HealthCare, USA) (Verdelis et al., 2011). The morphology of the barium-labelled $M \Phi$ was assessed by light microscopy and the effects of the uptake of barium sulfate particles on the metabolic activity of $\mathrm{M} \Phi$ were investigated with a watersoluble tetrazolium (WST-1) cell proliferation assay (Madison, WI, USA) (Mosmann, 1983).

\subsection{Mouse model of allergic asthma}

Female BALB/c mice (4-6 weeks old) were purchased from Harlan Laboratories and maintained with 'ad libitum' food and water. For generation of the experimental allergic airways disease model, mice were sensitized intraperitoneally (i.p.) at day 0 and 21 with $10 \mu \mathrm{g}$ ovalbumin (OVA), dissolved in $200 \mu \mathrm{l}$ PBS. At day 28 and 29, mice were treated intranasally (i.n.) with a solution of $100 \mu \mathrm{g}$ OVA/50 $\mu \mathrm{l}$ PBS to induce an acute allergic reaction (Biffi et al., 2013). Healthy age and gendermatched BALB/c mice, immunized and challenged with PBS only, were used as controls. To verify the success of OVA immunization, blood samples were taken $72 \mathrm{~h}$ after the last challenge from the facial vein of the living mice and levels of OVA-specific immunoglobulin within the sera were analysed as previously described (Biffi et al., 2013).

Animal in vivo procedures were performed at the CBM Animal Facility, Trieste, Italy, in compliance with the guidelines of the European (86/609/EEC), the Italian (DL116/92) and at the University Medical Center Göttingen, Germany, in accordance with the German ethical laws (33.9-42502-04-10/ 0134) and were approved by the Italian Ministry of Health as well as by the animal ethics administration of Lower Saxony, Germany.

\subsection{Application of macrophages and experimental setup}

Previous differential cell counts from bronchoalveolar lavages (BAL) and in vivo fluorescence measurement experiments showed that OVA-induced asthmatic mice display the strongest signs of inflammation between $48 \mathrm{~h}$ and $72 \mathrm{~h}$ after the last antigen challenge (Biffi et al., 2013). We therefore instilled $6 \times 10^{6}$ barium- and DiD-labelled M $\Phi$ resuspended in $30 \mu \mathrm{l}$ PBS intratracheally (i.t.) $72 \mathrm{~h}$ after the last OVA challenge into the lungs of asthmatic and control mice under xylazine-tiletamine-zolazepam anaesthesia.

\subsection{In vivo optical imaging}

Optical imaging was performed by two-dimensional fluorescence reflectance imaging (FRI) using the Optix MX2 system (ART; Montreal, Canada) as previously described (Markus et al., 2014). For in vivo scans, mice were anaesthetized by inhalation with isoflurane ( $2 \%$ isoflurane in 21 oxygen per min). Before imaging, mice were shaved and chemically depilated over thorax and abdomen to decrease scattering from the fur. Mice were scanned before, directly after and $24 \mathrm{~h}$ after $M \Phi$ instillation. All data were acquired using a $670 \mathrm{~nm}$ excitation laser diode in combination with a $700 \mathrm{Lp}$ emission filter and a $1.0 \mathrm{~mm}$ raster. The fluorescence 
intensity was analysed using the OptiView-2-02-00 software (ART). The average intensity of the lung area was measured and its relative increase compared with the pre-scan was computed.

\subsection{Preparation of biological samples for ex vivo $\mathrm{CT}$ analysis}

Mice were sacrificed $24 \mathrm{~h}$ after instillation of bariumsulfate-loaded and fluorescent-labelled M $\Phi$ (Fig. 1) using a xylazine-tiletamine-zolazepam overdose. In order to ensure comparability between different samples, all lungs were inflated in situ with air, under a constant pressure of $30 \mathrm{~cm}$ water column $(2.94 \mathrm{kPa})$ through a series of smaller tubes, which ended in a PE50 polyethylene cannula fixed inside the trachea with a cotton thread. Tracheas were tied up and all samples were kept at room temperature for $2 \mathrm{~h}$, in order to avoid any alterations caused by rigor mortis. To avoid air leakage, alterations and movements during the X-ray examination, samples were then embedded in a $1 \%$ agarose gel inside $30 \mathrm{ml}$ tubes (Fisher Scientific, USA) and kept for $30 \mathrm{~min}$ at $277 \mathrm{~K}$. Samples were moved to the synchrotron beamline $30 \mathrm{~min}$ before scanning to allow for temperature adaptation. Four lung samples were prepared for fXPCT analysis: one mouse with OVA-induced asthma and two healthy controls, all injected with $\mathrm{M} \Phi$ (AA, CN1 and CN2), as well as one healthy mouse without application of $\mathrm{M} \Phi$ which served as a negative control (Blk).

\subsection{Synchrotron-radiation-based $\mathrm{fXPCT}$ and phase retrieval}

All data sets were acquired at the SYRMEP beamline at the synchrotron light source Elettra (Trieste, Italy) (Brun et al., 2010; Dreossi et al., 2008), which is especially designed for medical applications and analysis of biological samples. The beamline was operated at $22 \mathrm{keV}$ at a sample-to-detector distance of $30 \mathrm{~cm}$. The central area of the lung of each sample was scanned by performing two overlapping $360^{\circ}$ scans with 1800 projections each and a $2 \times 2$ binning of the detector

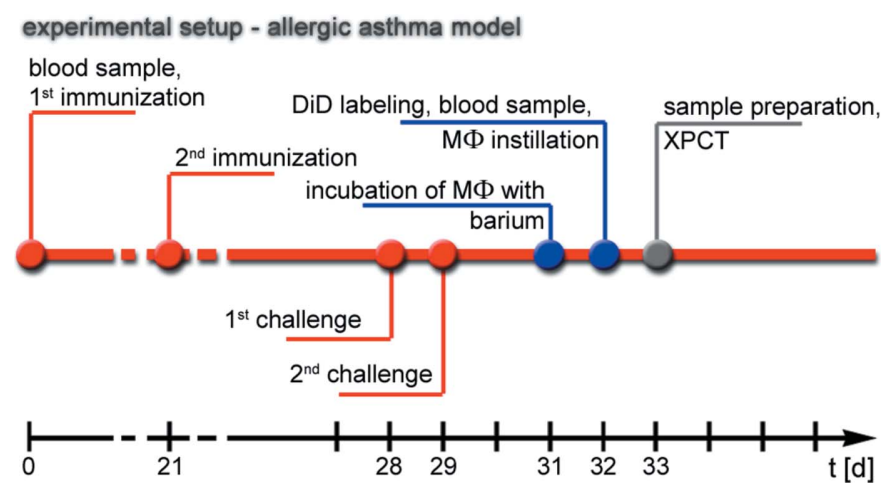

Figure 1

Experimental setup. An OVA allergen-induced experimental allergic airways disease model was used, consisting of two immunization steps (i.p. injection of $10 \mu \mathrm{g}$ OVA) on days 0 and 21 and two challenging steps (i.n. application of $100 \mu \mathrm{g}$ OVA) on days 28 and 29. Prior to their instillation, $M \Phi$ were labelled with Micropaque and $\mathrm{DiD}$ for 24 and $4 \mathrm{~h}$, respectively. On day $32,6 \times 10^{6}$ barium- and DiD-labelled M $\Phi$ were instilled i.t. into asthmatic and control mice. Mice were euthanized on day 33 and prepared for fXPCT analysis. elements, thus resulting in a spatial resolution of $9 \mu \mathrm{m}$. In order to decouple phase and absorption information in the acquired projection images, we applied a single-distance inline phase-retrieval algorithm based on the Born equation implemented by Chen et al., which only requires one data set, obtained at a single sample-to-detector distance (Mohammadi et al., 2014; Chen et al., 2010b, 2012; Taylor, 1981). In order to apply this class of algorithms, a priori knowledge of the $\delta$-to- $\beta$ ratio within the sample is needed. Within this study we used a $\delta$-to- $\beta$ ratio of 1950 for standardized lung tissue with a hydrogen, carbon and oxygen ratio: H10 C0.83 O5 (International Commission on Radiological Protection) (Mohammadi et al., 2014).

\subsection{Quantification of morphological alterations within the lung and distribution of barium-loaded macrophages}

For a quantitative comparison of the lung samples it is crucial to identify parameters that characterize the alterations in the lung structure without being affected by local inhomogeneities in the manifestation of the asthmatic reaction within the lung. To this end we applied an analysis scheme that was adapted from strategies used for the characterization of trabecular bone structure and other porous materials. Briefly, the data sets were reoriented and resampled to ensure that the analysis is independent of the orientation of the lungs during the scan. Due to the high contrast-to-noise ratio in the phaseretrieved reconstructed data sets, non-overlapping greyscale ranges were assigned to air, lung soft-tissue, bone of the rib cage and to barium used as label for the i.t. instilled $\mathrm{M} \Phi$. These image segments were then further analysed and quantified in terms of air, soft-tissue and barium content as well as narrowing of the airways.

\subsection{Histology}

Lung samples for histology were obtained from a further set of OVA-induced asthmatic and control mice following the schedule and conditions as described in Fig. 1 (in order to be comparable with the fXPCT analysis). The excised lungs were fixed in $10 \%$ buffered formalin and embedded in paraffin, and $3 \mu \mathrm{m}$-thick paraffin lung sections containing main stem bronchi were obtained. A periodic acid-Schiff (PAS) staining was performed to assess bronchial wall thickness and mucus production (Fullmer, 1960). Sections were deparaffinised, rehydrated and stained with periodic acid for $5 \mathrm{~min}$, followed by Schiffs reagent (Merck, Darmstadt, Germany) for 15 min and hematoxylin for $2 \mathrm{~min}$. Slices were washed for $3 \mathrm{~min}$ between each of the staining steps. The samples were dehydrated using an ascending alcohol series and Xylol and finally mounted with DePex (Serva, Heidelberg, Germany). An Axioskop 2 (Carl Zeiss Microscopy GmbH, Jena, Germany) microscope in combination with a Leica DC 100 camera (Leica, Switzerland) was used for analysis of the stained sections. 


\subsection{Fluorescence microscopy}

In order to verify the location of the injected DiD and barium-labelled $M \Phi$, fluorescence microscopy of lung tissue sections of asthmatic and control mice was performed, utilizing an Axiovert 200M inverted microscope (Carl Zeiss Microscopy) equipped with a xenon lamp and a high-sensitivity ORCA-AG digital camera (Hamamatsu, Japan), capable of NIRF detection. For this purpose, lungs of asthmatic mice $(n=2)$ and a control mouse, explanted $24 \mathrm{~h}$ after M $\Phi$ i.t. instillation, were filled with optimal-cutting-temperature (OCT) embedding material (Tissue-Tek; Sakura Finetek, Torrance, CA, USA) and were cryofrozen. Lung cryosections $(5 \mu \mathrm{m})$ were obtained, fixed with acetone $(10 \mathrm{~min}$ at $253 \mathrm{~K})$ and washed with tris(hydroxymethyl)-aminomethane (Tris) buffer ( $\mathrm{pH}$ 7.5). Immunostaining was performed as follows. Autofluorescence was blocked with $0.1 \mathrm{M}$ glycin [for $10 \mathrm{~min}$ at room temperature (RT)], endogenous biotin and avidin were blocked with Avidin Biotin Blocking Solution and unspecific binding sites for $20 \mathrm{~min}$ at RT with SEA BLOCK blocking buffer (both Thermo Scientific) following the manufacturer's protocol. Slices were then incubated overnight at $277 \mathrm{~K}$ with rat monoclonal anti-CD68 antibody (FA-11; Abcam, Cambridge, UK; $3.33 \mu \mathrm{g} \mathrm{ml}^{-1}$ ) diluted in Antibody Diluent with Background Reducing Components (DAKO, Glostrup, Denmark), followed by two incubation steps of $1 \mathrm{~h}$ at RT with biotinylated goat-anti-rat antibody (BioLegend, San Diego, CA, USA; 1:200), and with Streptavidin-AlexaFluor 555 (Molecular Probes, Life Technologies, Carlsbad, CA, USA; 1:400). Finally, slices were mounted with Mowiol (Calibiochem, Merck, Darmstadt, Germany) supplemented with 4',6-diamidino-2-phenylindole (DAPI) for staining of the nuclei and left overnight at $277 \mathrm{~K}$. Two washing steps with Tris buffer were performed between each step. DiD fluorescence was acquired using a band-pass (BP) $640 \pm 15 \mathrm{~nm}$ excitation filter, a $660 \mathrm{~nm}$ dichroic mirror and a BP $690 \pm 25 \mathrm{~nm}$ emission filter. The AlexaFluor 555 signals were recorded using a BP $546 \pm 6 \mathrm{~nm}$ excitation filter, a $580 \mathrm{~nm}$ dichroic mirror and a $590 \mathrm{~nm}$ long pass emission filter. DAPI fluorescence was acquired using a BP $365 \pm 12.5 \mathrm{~nm}$ excitation filter, a $395 \mathrm{~nm}$ dichroic mirror and a BP $445 \pm 25 \mathrm{~nm}$ emission filter. In the produced images the DAPI channel was set to blue, AlexaFluor 555 to green and DiD to red. Image generation and processing were performed using the software AxioVision Rel.4.6 (Carl Zeiss Microscopy GmbH, Jena, Germany) and ImageJ (National Institutes of Health, Bethesda, MD, USA) (Collins, 2007).

\subsection{High-resolution synchrotron-radiation-based X-ray phase-contrast microCT (HR microCT)}

Lung sections of a third set of OVA-induced asthmatic mice $(n=2)$ and a control mouse following the schedule and conditions as described in Fig. 1 were also studied using a CT set-up with higher resolution. For this aim the procedure for sample preparation was the following: the trachea was cannulated, then filled and fixed with $4 \%$ paraformaldehyde (PFA), and subsequently single lobes of the lung were embedded in a 5\% agarose-gel. Finally, $500 \mu \mathrm{m}$-thick slices were cut using a Vibratome (Leica VT 1000S; Leica, Switzerland). The slices were placed with a droplet of PBS between two round pieces of polypropylene foil, secured within aluminium rings. These were glued together to create a closed chamber for the slices (Olendrowitz et al., 2012). These slices were then imaged with a divergent X-ray beam of $17.5 \mathrm{keV}$ at the beamline ID22 $\mathrm{Ni}$ at the European Synchrotron Radiation Facility (ESRF) in Grenoble. The beam was focused to less than $100 \mathrm{~nm} \times 100 \mathrm{~nm}$ by Kirkpatrick-Baez (KB) mirrors and free propagation phase-contrast images recorded with a FReLoN camera (Analog and Transient Electronic ESRF group) coupled to a scintillator (Weitkamp et al., 1999). The sample was placed at the maximum possible defocus distance to achieve the highest field of view. Using a ten-fold objective lens behind the scintillator and the magnification of the KB beam an effective pixel size of $430 \mathrm{~nm}$ was achieved. Tomographic scans with a series of 1500 images over a full rotation of $360^{\circ}$ were recorded for several specimens of asthma and control mice. Phase retrieval was performed using the single-distance Holo-tomo reconstruction algorithm (Cloetens et al., 1999) implemented at the beamline. Before $3 \mathrm{D}$ reconstruction, each projection image was corrected by an image of the empty beam and aligned to the other projections before phase retrieval was performed.

\subsection{Analysis and statistics}

The 3D rendering of the data sets was performed with VGStudio Max 2.2 (Volume Graphics, Heidelberg, Germany). For mask generation, IDL 7.0 (Research Systems; Boulder, $\mathrm{CO}$, USA) and ImageJ (National Institutes of Health; Bethesda, MD, USA) were used (Abràmoff et al., 2004). Pore3D, a proprietary software library developed by the SYRMEP group, was applied to analyse the 3D barium distribution as well as to quantify the air and tissue content of the lung within volumes of interest (VOIs) (Brun et al., 2010). Statistical analysis was performed using MINITAB (Minitab Ltd; Coventry, UK) and utilizing a one-way ANOVA test with Tukey $90 \%$ simultaneous confidence intervals for the computed parameters (Ryan et al., 2005; Dunn, 1961).

\section{Results}

\subsection{XPCT in combination with single-distance phase retrieval is a valuable tool for lung imaging}

In order to evaluate the usefulness of phase retrieval in our experiment, we performed two scans of a mouse lung sample in XPCT at sample-to-detector distances of 7 and $30 \mathrm{~cm}$. At the distance of $7 \mathrm{~cm}$ only minor edge effects were present and therefore this essentially resembles the absorption-based setup in classical CT, despite the fact that the scan was performed with a monochromatic X-ray source. In contrast, scanning the same sample at a sample-to-detector distance of $30 \mathrm{~cm}$ provides sufficient phase contrast. Phase retrieval was applied to the projection images of the second scan before $3 \mathrm{D}$ reconstruction with FBP was performed. This procedure 
matches the same scheme used in this study. As a basis for a quantitative comparison, we calculated the contrast-to-noise ratio (CNR) using the following equation:

$$
\mathrm{CNR}=\frac{\overline{g_{1}}-\overline{g_{2}}}{\left[\left(\sigma_{1}^{2}+\sigma_{2}^{2}\right) / 2\right]^{1 / 2}},
$$

where $\overline{g_{1}}$ and $\overline{g_{2}}$ denote the mean grey value of two adjoining tissues and $\sigma_{1}^{2}$ and $\sigma_{2}^{2}$ reflect their noise level, measured as squared standard deviation in a region of interest (ROI) (Mohammadi et al., 2014). In each sample and on five reconstructed slices homogeneously distributed over the whole lung, eight circular two-dimensional ROIs (size $\sim 0.4 \mathrm{~mm}^{2}$ ) solely containing either air or lung soft-tissue were defined and analysed. We determined a CNR between air and lungtissue of about 20.0 in the phase-retrieved data set at $30 \mathrm{~cm}$ and 1.9 at $7 \mathrm{~cm}$ without PhR. In addition, only minor blurring of the phase-retrieved images was found (data not shown). These results demonstrate that the combination of XPCT with single-distance phase retrieval is able to increase the softtissue contrast in our samples and setup by a factor of 9.8 when compared with absorption-based monochromatic X-ray imaging. This directly translates into an increased sensitivity that is beneficial for the combined functional and structural CT imaging approach to visualize mouse lung tissue.

\subsection{Macrophages as specific contrast agent for functional CT}

The barium sulfate uptake efficacy of $M \Phi$ was assessed with a bench-top microCT. Imaging of a vial, containing $1 \times 10^{5}$ M $\Phi$ loaded with barium sulfate particles and resuspended in $100 \mu \mathrm{l}$ PBS, showed a $10 \%$ increase of the X-ray absorption when compared with a vial containing $1 \times 10^{5}$ unlabelled $\mathrm{M} \Phi$ as control (data not shown). The WST-1 assay revealed no influences of the phagocytized barium sulfate particles on the metabolic activity of the MФ (data not shown). Furthermore, no evidence of morphologic alterations was observed in barium-sulfate-loaded $M \Phi$ by light microscopy (data not shown). In conclusion, the approach to load $M \Phi$ with barium provides sufficient contrast for CT imaging and shows no signs of acute cell toxicity.

\subsection{In vivo optical imaging demonstrates successful intra- tracheal instillation of $\mathrm{M \Phi}$}

In vivo NIRF imaging was performed in order to confirm the successful i.t. instillation of the $M \Phi$. For this purpose the M $\Phi$ were additionally stained with the NIR cell membrane label DiD. Fig. 2 shows the in vivo optical imaging results for the same asthmatic mouse analysed later by fXPCT. A strong increase in fluorescence intensity was observed over the lung area directly and $24 \mathrm{~h}$ after i.t. instillation of DiD- and bariumlabelled $M \Phi$ when compared with the pre-scan. These results verify the presence of the labelled $M \Phi$ in the lung area of the mouse $24 \mathrm{~h}$ after i.t. administration.

\subsection{Processing and quantification of fXPCT data sets}

All lungs were kept in situ and scanned at the SYRMEP beamline using a setup that allows for fXPCT. In order to cover the main central area of the lung, two slightly overlapping scans per sample were performed. The original projection data sets were then processed by the single-distance in-line phase-retrieval algorithm (Chen et al., 2010) to create projections predominately showing the $\delta$ part of the complex refractive index. These data sets were later on reconstructed with FBP. In order to analyse the data quantitatively, the following steps were performed: stitching of the two overlapping scans and reorientation of the data sets to allow for comparison between different samples; masking of the lung to restrict the analysis to the lung area; segmentation of the three different components (air, lung soft-tissue and barium); 3D quantification of structural alterations and depiction of the barium concentration and distribution.
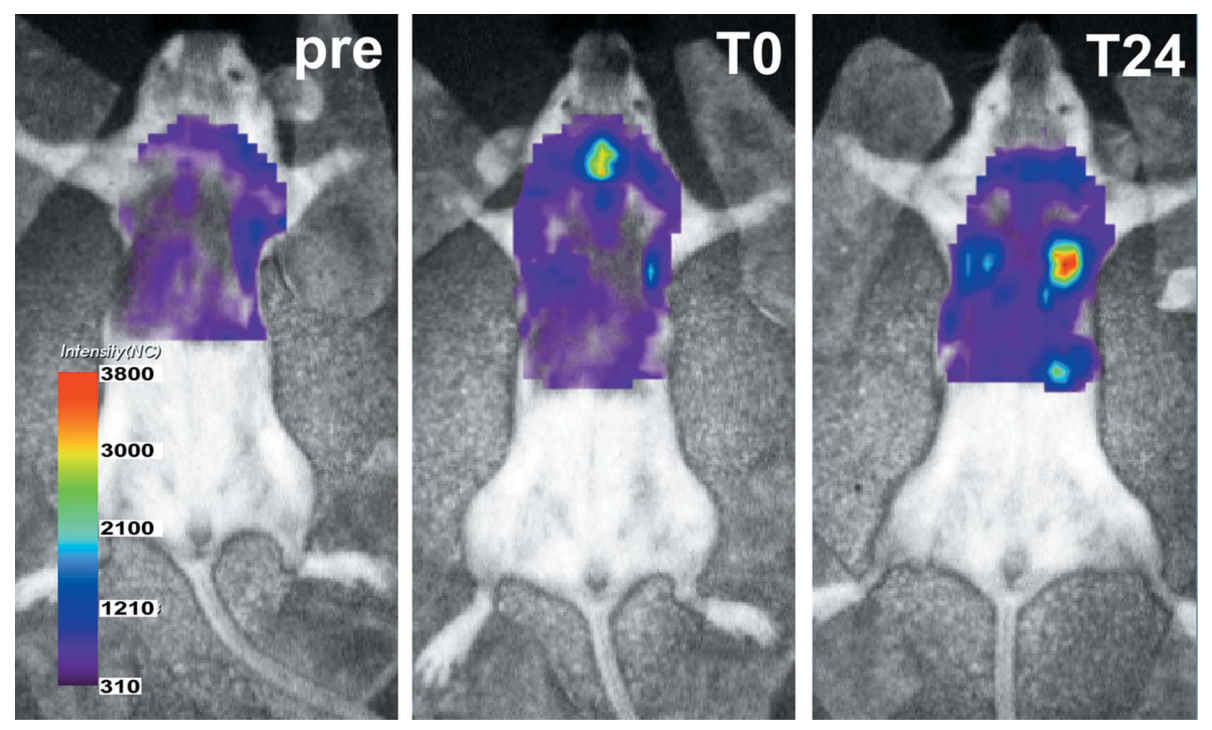

Figure 2

In vivo optical imaging results. Fluorescence intensity maps of the same asthmatic mouse used for fXPCT (Fig. 3) before (pre), directly after (T0) and $24 \mathrm{~h}$ after (T24) i.t. instillation of $6 \times 10^{6}$ barium-sulfate-filled and DiD-labelled M $\Phi$ are shown. After $24 \mathrm{~h}$, strong fluorescence signals expressed in normalized counts (NC) are visible over the lung area. 


\subsubsection{Post-processing steps and descriptive comparison of} the obtained IXPCT lung data sets. The automatic quantification of anatomical alterations is based on the splitting of the data sets into rectangular VOIs. Thus, to ensure that all data sets are present in the same orientation, the reconstructed data of the two scanning steps were registered, combined and re-orientated. In consideration of the memory limitations in the post-processing algorithms, data sets were re-sampled down to an isotropic resolution of $14.4 \mu \mathrm{m}$. A volumerendering representation of the final data sets is shown in Fig. 3(a) and VOIs in Figs. 3(b) and 3(c). To visualize the various tissues as well as the i.t. instilled barium-sulfate-loaded $\mathrm{M} \Phi$, pseudo-colours were assigned to different greyscale ranges. As a result, lung soft-tissue is displayed in red, bones in grey and highly dense areas related to the barium-containing M $\Phi$ appear yellow. In order to maintain the 3D visibility of the inner lung structure the air was set to transparent. Given that barium causes a greater phase shift than lung soft-tissue and the healthy blank (Blk) contains no barium, the upper limit of the grey value range representing the lung soft-tissue in Blk was chosen as a valuable threshold to detect the bariumloaded MФ.
The segmented images clearly show an increased soft-tissue content in sample AA in comparison with the healthy controls [Figs. 3(a) and 3(b)]. Additionally, the VOI of AA (Fig. 3b) displays a reduced porosity, which illustrates the airway obstruction characteristic for asthma. The same effect of increased soft-tissue content and airway obstruction can to a certain extent also be seen in the planar slices in Figs. 4(a) and 4(b) (white arrow heads). Furthermore, Fig. 3 demonstrates that the barium-sulfate-loaded $M \Phi$ seem to be distributed in cluster-like structures throughout the asthmatic lung, which is also demonstrated in planar reconstructed slices in Figs. 4(c) and $4(d)$. High-contrast regions related to barium-sulfateloaded $M \Phi$ are also present in the lungs of the two healthy controls $\mathrm{CN} 1$ and $\mathrm{CN} 2$; however, at a much lower quantity (yellow spots, Fig. $3 b$ ). Note that $\mathrm{M} \Phi$ derived signals within the lung in AA appear to be surrounded by lung soft-tissue and therefore seem to be originating from areas around the bronchial walls and not from the lumen of the airways (Fig. 3b). The same location of $\mathrm{M} \Phi$ was also found in twodimensional slices as shown in Fig. 4(c). In the same slices areas can be found that are solely composed of soft tissue that is characterized by a lower contrast than the marked spots

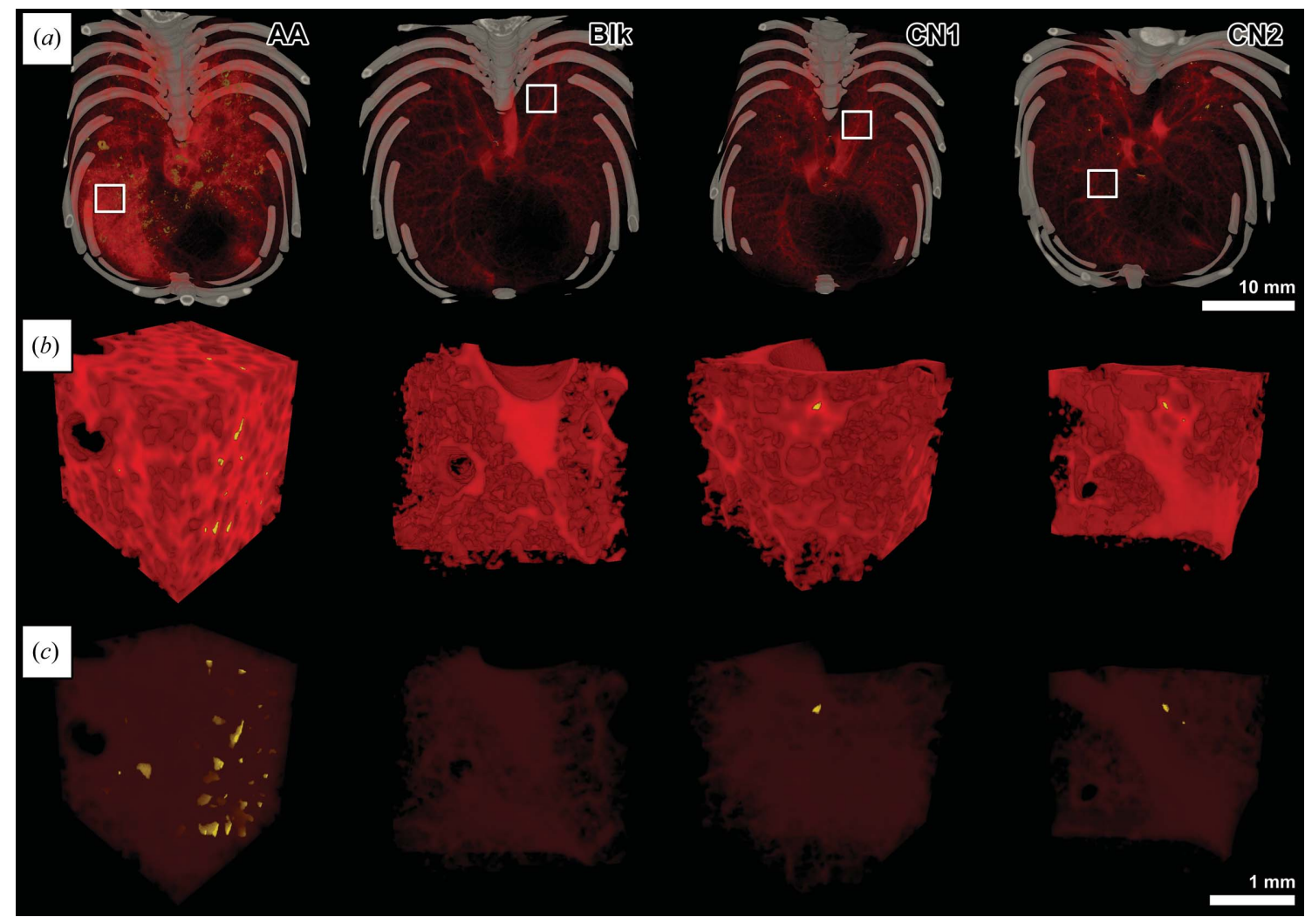

Figure 3

Volume rendering of the fXPCT results and visualization of the MФ localization. Volume renderings of four 3D reconstructions of phase-retrieved fXPCT data sets are shown. Each column represents one lung sample of an asthmatic mouse (AA), a healthy mouse without instilled M $\Phi$ as negative control (Blk), and two healthy controls $(\mathrm{CN} 1, \mathrm{CN} 2)$. Bone is displayed in grey, soft-tissue in red and barium in yellow (air is not depicted). (a) Illustration of the entire field of view: more barium and an increased soft-tissue density is shown in AA. (b) VOIs (their origin is indicated by white squares in row 1 ). (c) The same VOIs are shown but with higher transparency to allow visualization of the sites where barium-filled $M \Phi$ cluster in more detail. Note that in the VOIs of the AA lung a higher barium content is visible compared with the results obtained in lungs of the negative control Blk and the healthy controls CN1 and CN2. Additionally, the VOIs of the AA sample show a higher soft-tissue content in comparison with the VOIs of all controls, reflecting a reduced pulmonary volume and increased soft-tissue content in the asthmatic lung tissue. 
assigned to barium. Therefore, it can be excluded that the detected spots are caused by partial-volume effects. Due to the fact that no other strong absorbing material is present in the lung, these high-dense spots most likely represent the instilled barium-labelled $M \Phi$. Therefore, the detected localization of the $M \Phi$ within the bronchial walls may indicate an active migration of the instilled $M \Phi$ from the airspace into the tissue.

3.4.2. Development of an image-processing scheme for automatic 3D quantification of morphological alterations. Since lung tissue has a tree-like structure of airways with different capillarity, the depiction of the VOIs are of crucial importance for a VOI-based analysis scheme. On the other hand, the instilled barium-labelled $M \Phi$ produce a weak signal and might have an inhomogeneous distribution in such a way that they could be easily missed in an approach focused on the entire lung. In order to perform a quantitative comparison, entire lungs were therefore subdivided into three sets of $2 \mathrm{~mm}$-thick bands in the horizontal, vertical and frontal direction. In the so-generated 32 VOIs per sample, two types of parameters were analysed: (i) volume ratio (Vol. Ratio), which defines the volume fraction of a tissue or material of interest compared with the total volume of a VOI, and (ii) structural thickness (St. Th.) of either the airways or the surrounding tissue. Structural thickness is calculated by analysing the maximal size of spheres which can be inscribed in the structure as proposed by Hildebrand \& Ruegsegger (1997) and implemented in the software Pore3D (Brun et al., 2010). These two parameters are directly related to airway wall thickening and airway obstruction and therefore reflect structural changes characteristic for asthmatic lung tissue.

3.4.3. Quantification of morphological changes in the lung. In addition to visual inspection of the rendered fXPCT data sets, quantification of structural changes was a further aim of the study. For this purpose the average and standard variation of the parameters Vol. Ratio and St. Th. were calculated in each sample and in each of the horizontal, vertical and transvertical sets of VOIs, respectively (Table 1). We found an approximately $17 \%$ reduced air content as well as a $61 \%$ increase in the soft-tissue Vol. Ratio in the AA sample compared with all controls (Table 1). In addition, airway obstruction as a hallmark of asthma is clearly reflected in the results of the St. Th. measurements showing a $32 \%$ St. Th. reduction of the mean airway thickness in AA compared with all healthy animals and, vice versa, a $48 \%$ higher St. Th. in the lung softtissue (Table 1).

In order to prove whether the results obtained in the individual VOIs of each data set are significantly different in between the samples, we used a oneway ANOVA test with Tukey $90 \%$ simultaneous confidence intervals to test the difference of the mean values of the analysed parameters (Vol. Ratio of air and soft-tissue and St. Th. of airways and tissue) (Ryan et al., 2005). The comparison of AA and Blk revealed reliable differences for all analysed parameters. Even the minor differences of these parameters between Blk, CN1 and $\mathrm{CN} 2$ were found to be statistically significant, demonstrating the sensitivity of our fXPCT approach. Therefore, based on the chosen parameters (air and soft-tissue volume ratio and mean airway and soft-tissue thickness), the asthmatic and the three control lung samples can be successfully distinguished, indicating that this parameter set can be used to preclinically monitor structural changes in asthmatic lungs. 
Table 1

Mean values \pm standard deviation of the considered parameters.

The pooled standard deviations for the Vol. Ratio (\%) parameter were \pm 0.04 for barium, \pm 4.95 for alveoli and 4.94 for tissue. For the St. Th. $(\mu \mathrm{m})$ parameter the pooled standard deviation were \pm 68 for alveoli and \pm 11 for soft tissue.

\begin{tabular}{lllll}
\hline & Sample & Barium & Air & Soft tissue \\
\hline Vol. Ratio (\%) & AA & $0.10 \pm 0.07$ & $64.62 \pm 6.78$ & $35.28 \pm 6.75$ \\
& Blk & $0.00 \pm 0.00$ & $78.32 \pm 3.43$ & $21.68 \pm 3.43$ \\
& CN1 & $0.00 \pm 0.00$ & $74.28 \pm 4.75$ & $25.72 \pm 4.75$ \\
& CN2 & $0.00 \pm 0.00$ & $81.85 \pm 4.21$ & $18.15 \pm 4.21$ \\
St. Th. $(\mu \mathrm{m})$ & AA & & $188 \pm 39$ & $83 \pm 15$ \\
& Blk & & $254 \pm 53$ & $54 \pm 9$ \\
& CN1 & & $256 \pm 83$ & $60 \pm 9$ \\
& CN2 & & $315 \pm 87$ & $53 \pm 7$ \\
\hline
\end{tabular}

In addition, the histogram of the $\delta$ distribution within the samples, which was normalized to the total amount of analysed voxels for each mouse, showed a more dominant interval in AA (grey values 45-55, data not shown). This result suggests that during the course of asthma not only structural but also changes in the composition of the lung tissue occur, which may be the result of the presence of oedema, an increased mucus production or alterations in the collagen fibres within the lung.

3.4.4. Quantitative analysis of the barium concentration within the lungs. As shown in Fig. 3 (in yellow), barium-filled $\mathrm{M} \Phi$ can be detected in AA and to a lower extent in $\mathrm{CN} 1$ and $\mathrm{CN} 2$. Following the same scheme used to quantify the softtissue content, but applied to the barium segment of the data set, we found a Vol. Ratio of about $0.1 \%$ for barium in the AA lung (Table 1). The barium concentration in the two healthy controls $\mathrm{CN} 1$ and $\mathrm{CN} 2$ was below 1\%o and therefore appear as 0 in Table 1 . The Blk mouse that did not receive bariumfilled $\mathrm{M} \Phi(\mathrm{Blk})$ was used to set up the threshold and consequently shows no barium content within the whole lung. The Tukey intervals for barium content (data not shown) are all positive and therefore confirm that the Vol. Ratio of the barium content is significantly larger in the AA lung sample than in the Blk and $\mathrm{CN}$ mice.

In summary, bronchial wall thickening and airway obstruction in asthmatic mouse lung tissue can be visualized in fXPCT data sets [Figs. 3, 4(a) and 4(b)]. Instilled M $\Phi$ appear within clusters around the bronchial walls [Figs. 3, 4(c) and $4(d)$ ]. With our approach we can also quantify these alterations using automatically generated 3D morphologic parameters. These parameters, i.e. Vol. Ratio of air, barium and lung soft-tissue and St. Th. of air and lung soft-tissue, showed significant differences between AA and healthy controls in accordance with the known pathological features in lungs of an asthma mouse model.

\subsection{Fluorescence microscopy and HR microCT confirm the accumulation of $M \Phi$ in asthmatic lung tissue}

In order to confirm the accumulation and to further analyse the location of the instilled barium-filled and DiD-labelled M $\Phi$ within the lung observed by fXPCT, we performed fluorescence microscopy and HR microCT on lung sections.
Since the preparation process and the analysis at the SYRMEP beamline resulted in the deterioration of the samples and excluded histological analysis, fluorescence microscopy and HR microCT were performed with lungs of a different cohort of mice, but strictly following the same protocol for asthma induction and instillation of $\mathrm{M} \Phi$ (Fig. 1), including the use of the same batch of OVA.

Representative results of the fluorescence microscopy performed on cryosections of a lung of an asthmatic mouse (AA) and a control lung (CN) are shown in Figs. 5(a) and 5(b). Instilled barium- and DiD-labelled $M \Phi$ are shown in red, DAPI stained nuclei appear blue and an anti-CD68 antibody (Ab) which binds to both the applied and endogenous $M \Phi$ is shown in green. As a result, the instilled barium- and DiDlabelled MФ appear yellow in the merged image. We observed DiD-labelled M $\Phi$ in the small alveoli [Figs. 5(a) and 5(b), white arrows] in both the $\mathrm{CN}$ and the AA lung samples. As already seen in the fXPCT analysis, more instilled M $\Phi$ are present in the AA lung. Moreover, only in the asthmatic tissue were we able to detect an accumulation of instilled M $\Phi$ within lung areas of high cellular density [Fig. 5(a), detailed view]. These clusters of cells were found throughout the lung and in close proximity to the bronchi. Additionally, a higher number of endogenous $\mathrm{M} \Phi$ (green) was found in the asthmatic lung [Fig. 5(a), green staining].

HR microCT examination clearly verified the presence of the barium-filled $\mathrm{M} \Phi$ inside the lung (dark spots), as shown in Figs. 5(c) and 5(d), for two lung sections from an asthmatic and a control mouse, respectively. In both asthmatic and healthy lungs, barium-filled $\mathrm{M} \Phi$ were detected throughout the lung tissues. However, in contrast to the healthy sample, the asthmatic sample additionally displayed areas around the bronchi with an increased accumulation of M $\Phi$ (white rectangle). Furthermore, thickening of the bronchial walls was observed in the asthmatic tissue [Fig. 5(c), white arrow head] compared with the healthy control [Fig. $5(d)$ ], reflecting the presence of the disease.

Figs. 5(e) and 5(f) show PAS-stained histological slices from lungs of an OVA-induced asthma mouse (AA) and a healthy control $(\mathrm{CN})$. Red dots indicate that mucus production is solely present in the asthmatic lung (Fig. 5e). Furthermore, airway wall thickening can be observed (black arrow head) in the AA slide only. These findings represent typical characteristics of asthma and confirm the presence of an acute asthma reaction in these mice. In addition, ELISA analysis of the sera revealed increased IgG1 titre for all asthma mice (data not shown), verifying a successful immunization.

\section{Discussion}

This study presents a novel functional in-line free propagation X-ray phase-contrast CT imaging approach (fXPCT) that enables the depiction of both structural features of lung tissue and the accumulation of barium-labelled $M \Phi$ in the lungs of mice after intratracheal instillation. We show that fXPCT can be applied for CT-based immune cell tracking studies. Furthermore, this method allows the quantification of struc- 
tural changes in whole asthmatic lungs in situ by measuring parameters like the volume ratios of air, soft-tissue and the mean airway and soft-tissue thickness, thereby assessing hallmarks of asthma such as bronchial wall thickening and airway obstruction.

By applying a single-distance phaseretrieval algorithm (Paganin et al., 2002) to decouple phase from absorption information, we raised the CNR of lowabsorbing unstained lung soft-tissue by a factor of ten when compared with classical FBP (Mohammadi et al., 2014; Chen et al., 2010). It has to be emphasized that this factor is strongly related to the experimental setup used, including the sample-to-detector distance, the type of samples, the pixel size of the camera system, the quality of the X-ray beam, the phase-retrieval and reconstruction algorithms, and does therefore not represent a general rule for comparing phase-retrieved with non-phase-retrieved CT reconstructions (Donnelly et al., 2003). In a different setup even a factor of up to 200 was reported by Beltran et al. (2011). Another way to improve the detectability of contrast agents would be $K$ edge imaging (Cormode et al., 2010), in spite of the increase in sensitivity in $K$-edge imaging in combination with a non-energy-resolved detector requiring two scans with at least one using a photon energy higher than optimal for soft-tissue contrast.

Single-distance phase retrieval uses, as the name implies, only one projection to calculate the $\delta$-distribution of the refractive index. Therefore, it simplifies the experimental setup and minimizes the acquisition time. This is of particular advantage for unfixed biological samples as used in this study, as it reduces the influence of alterations that occur within the samples over
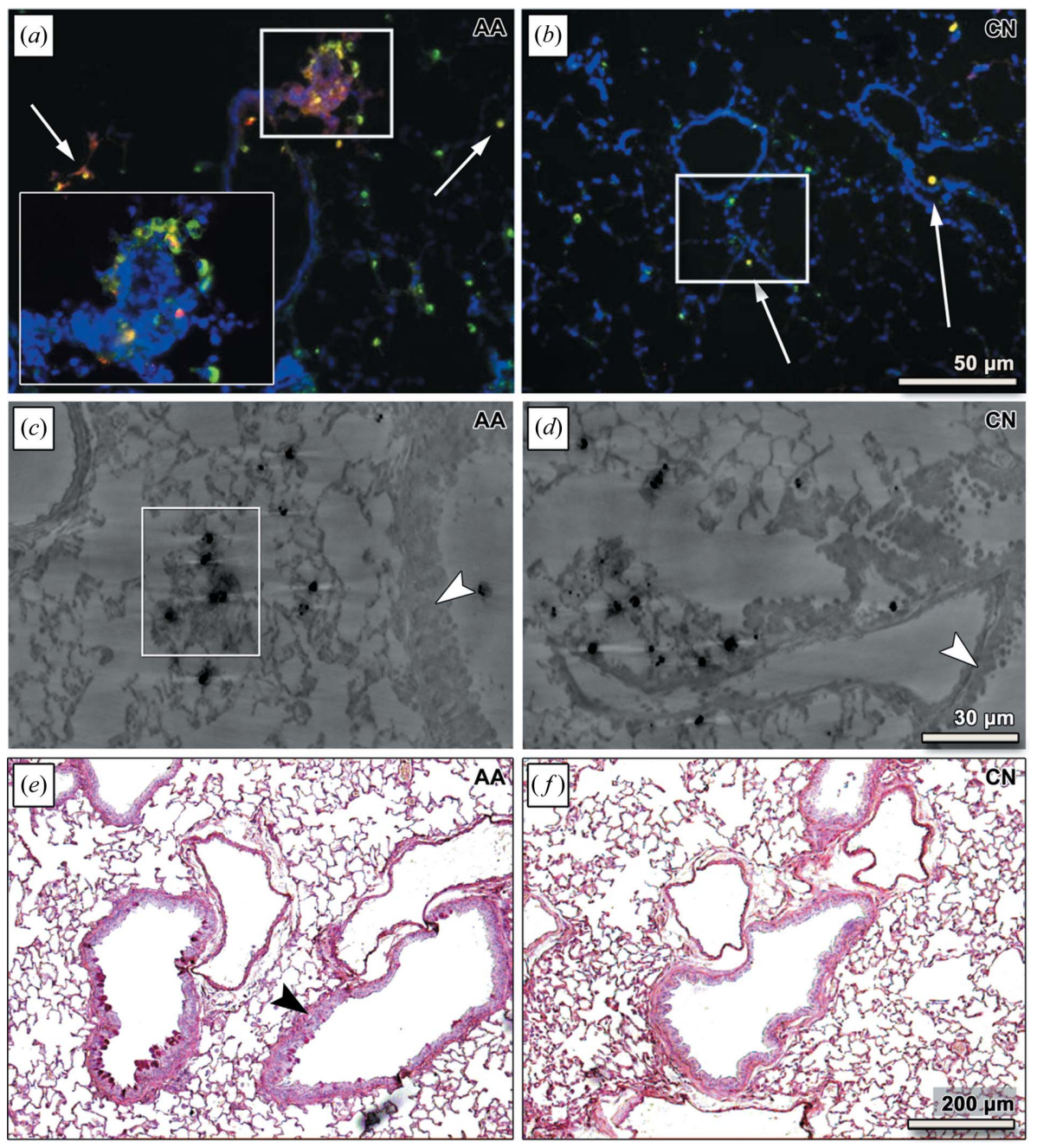

\section{Figure 5}

Validation of the fXPCT results by fluorescence microscopy, HR microCT and histology. $(a, b)$ Fluorescence microscopy of lung sections of $(a)$ an asthmatic (AA) and $(b)$ a control mouse $(\mathrm{CN})$. Nuclei were stained with DAPI (blue) and $M \Phi$ were immuno-stained with an anti-CD68 antibody (green). DiD-labelled $M \Phi$ are shown in red, whereas instilled $M \Phi$ appear yellow due to the double staining with CD68 and DiD. Endogenous M $\Phi$ are shown in green and can be seen in alveoli of both AA and CN lungs, but seem to form clusters in AA ( $a$ and $b$ ). Instilled DiD-labelled M $\Phi$ are also visible in both $\mathrm{AA}$ and $\mathrm{CN}$ lung samples (white arrows). In contrast to the $\mathrm{CN}$, the AA sample shows high cellular density areas (white rectangle and detailed view), where several CD68 positive cells and clusters of instilled $\mathrm{M} \Phi$ can be observed. Unlike the AA sample, the $\mathrm{CN}$ does not show these areas, which may explain the increased soft-tissue ratio and the airway obstruction found in the fXPCT results. $(c, d)$ Representative cross sections of $\mathrm{HR}$ microCT for AA and $\mathrm{CN}$ vibratome lung section. An increased wall thickness (white arrow head) for AA $(c)$ and the location of the labelled $\mathrm{M} \Phi$ (black dots) can be clearly depicted. $\mathrm{M} \Phi$ can be seen in both $\mathrm{CN}$ and AA, $(c)$ shows an area with higher cell density and accumulation of $M \Phi$ (white rectangle). ( $e$ and $f$ ) PAS-stained lung sections of an asthmatic (AA) and a control mouse (CN). Increased wall thickness in asthmatic lung tissue (black arrow head) is depicted. Red dots are exclusively visible in asthmatic lungs and depict mucus production.

time. However, single-distance phase retrieval is in principle based on the assumption that the studied sample is a 'homogeneous' object, meaning it only contains one known $\delta$-to- $\beta$ ratio (Chen et al., 2011; Gureyev et $a l ., 2009)$. Since this is not the case for our biological samples, a $\delta$-to- $\beta$ ratio optimized for lung tissue was used. Lung is only composed of low- $Z$ materials $(Z<10)$ for which Wu et al. (2005) showed already that single-distance phase-retrieval algorithms can be applied. Nevertheless, the generated reconstructions are not valid for the analysis of dense struc- tures like bony components (Beltran et al., 2011). If the skeleton is of interest, an additional phase-retrieval step in combination with a different $\delta$-to- $\beta$ ratio should be applied as shown by Beltran et al. (2011). Here we consider a low concentration of barium-sulfate-loaded $M \Phi$ (less than $0.2 \%$ ) only a minor disturbance of the assumed 'homogeneity' within the lung although barium has a $Z$ value of 56 . The calculated ten-fold increase in CNR is based on the comparison with a scan that uses the same setup, but with a 
minimized sample-to-detector distance, generating projection images with predominately absorption-based contrast. Therefore, the reconstructed absorption-based data set was acquired with quasi-monochromatic X-rays known to generate higher-quality data than conventional microCTs with the same spatial resolution (Sera et al., 2005). Thus, the gain in contrast with single-distance phase retrieval is even more prominent when compared with conventional benchtop microCT scans. This increase in contrast directly leads to an increased sensitivity, which in our case did not only enable the depiction but also the quantification of parameters of structural changes in the entire asthmatic lung in 3D, namely the volume ratios of air, soft-tissue and the mean airway thickness, and was the prerequisite for the detection of the labelled $\mathrm{M} \Phi$.

In contrast to other studies that analyse mouse lung structure at a micrometre scale (Yong et al., 2009; Sera et al., 2005; Kitchen et al., 2005), we kept the lung in situ by filling the airways with air at a constant pressure. We believe that in this way the shape of the lung, which is critically dependent on the pressure and the boundaries given by the ribcage, can be compared with an in vivo situation. In previous studies using the same asthma mouse model and sample preparation scheme, we found that the overall lung volume (soft-tissue and air content) was greater in asthmatic than in the healthy mice. That means that the asthmatic lungs can be more inflated at the same pressure pointing to a change in the elasticity of the lung tissue. This finding may be reflected in the altered $\delta$-value distribution of the soft-tissue in the asthmatic sample and supports the understanding that in asthma, in addition to inflammation, changes in the composition of lung tissue occur, that lead to a loss in elastic recoil. This loss of elasticity was already observed in patients (Gelb \& Zamel, 2002; Gelb et al., 2002) and confirmed our previous studies.

With this study we present a novel set of parameters [volume ratio (Vol. Ratio) and structural thickness (St. Th.) of airways and soft-tissue] and show their potential use in describing differences between asthmatic and healthy lung tissue. By applying fXPCT we determined a $17 \%$ reduced air content, a $32 \%$ reduced mean airway thickness and a $61 \%$ increase in the soft-tissue content in the asthmatic lung tissue compared with controls, demonstrating that in contrast to histology or conventional CT our approach can precisely measure structural alterations and illustrate them in 3D. The inflammatory response in asthma eventually leads to bronchial obstruction, caused by structural abnormalities such as hypertrophy of airway smooth muscle, sub-epithelial fibrosis, goblet cell hyperplasia, and proliferation of airway blood vessels and nerves. Since routinely most of these morphological changes of airway remodelling are visualized histologically in lung sections in both humans and mouse models ( $\mathrm{Al}$ Heialy et al., 2011; Blacquière et al., 2010; Leong \& Huston, 2001; Epstein, 2004), the quantification of these changes has been difficult to date. While there are limited approaches by conventional CT and MRI, these techniques are restricted by a low resolution. In particular, minor alterations of the lung tissue in asthma cannot be visualized with the limited spatial resolution of clinical CT scanners. Therefore, up to now the diagnosis of asthma in $\mathrm{CT}$ is based on lung densitometry measuring air trapping caused by disturbed ventilation of the lung (Washko et al., 2012). Our approach provides novel parameters which may used in both preclinical asthma models and clinical practice to classify and monitor asthma of different severity and/or to access the influence of an asthma therapy on airway remodelling.

In addition to anatomical information, the increased sensitivity of our fXPCT technique in comparison with conventional microCT allows for the depiction of the distribution of i.t. instilled barium-sulfate-loaded immortalized alveolar macrophages as 'physiological' contrast agent within the 3D lung structure. This, to our knowledge, represents the first approach of using barium-sulfate-loaded macrophages as contrast agents for CT-based cell tracking studies in an experimental allergic airways disease model. So far the use of synchrotron-radiation-based CT for cell tracking has only been reported in a few applications such as imaging small clusters of tumour cells $e x$ vivo loaded with gold nanoparticles (Astolfo et al., 2013). The visualization of pancreatic islet cells encapsulated in barium microcapsules is another example for CT-based cell tracking (Arifin et al., 2012). We found a higher barium content in the asthmatic lung sample, which is most likely due to a more clustered distribution of the instilled macrophages in cell-dense areas around the bronchi, a finding which needs further investigation by high-resolution tomography. This result is supported by our validation experiments with fluorescence microscopy and HR microCT, all techniques that share a higher spatial resolution than the used fXPCT approach. In our experiment in asthmatic lung tissue, $24 \mathrm{~h}$ after instillation, $\mathrm{M} \Phi$ were predominately found in areas with an increased cell density. While the implications of these findings for the pathomechanism of asthma and other lung disease models require further investigation, our results demonstrate the benefit of our imaging technique for 3D localization of cells in specific tissue regions. As the M $\Phi$ used in this study are derived from an immortalized cell line (Mbawuike \& Herscowitz, 1989), no conclusions towards a similar behaviour of endogenous $\mathrm{M} \Phi$ can be made.

A current drawback of our method for future clinical application is the lack of clinical CT systems that provide phase contrast. This limitation may soon be overcome by the implementation of novel technologies such as liquid-metal jet anode systems, miniaturized synchrotrons or grating-interferometer-based phase-contrast imaging utilizing classical X-ray sources (Pfeiffer et al., 2006; Tompkins et al., 1998; van Heekeren et al., 2011). All of these techniques perform well under laboratory conditions but struggle with different technical problems, which limit their use in the clinic so far. In particular, the high radiation dose for grating-based phasecontrast devices hamper their clinical application at the moment (Raupach \& Flohr, 2011). However, our quantification scheme for analysis of lung structure alterations can be directly translated to any other CT or anatomical lung imaging technique, providing a spatial resolution high enough to resolve the lung substructure. 
Our approach presents a proof-of-principle study for fXPCT imaging that specifically visualizes and quantifies morphological differences and airway remodelling of the mouse lung and will support the preclinical validation of newly developed targeted diagnostics and drug delivery strategies for lung diseases. Furthermore, our novel imaging approach provides a solid system for cell tracking studies of immune cells to investigate the role that macrophages might play in the development and progression of lung diseases such as asthma.

This study was conducted within the European project Public Private Partnership for Asthma Imaging and Genomics (P3AGI, http://www.p3agi.eu) funded by the European Commission through FP7-IAPP Marie Curie Action (GA 230739) and was further funded by the Deutsche Forschungsgemeinschaft (DFG, DU 1403/1-1) and the EXTREMA COST action MP1207. Histology and optimization of the macrophage cultivation and labelling approach as well as preliminary microCT analysis was performed at the University Medical Center, Göttingen, Germany, with excellent technical assistance from Sarah Greco, Bärbel Heidrich and Rosi Streich. The beamline experiments were performed at the SYRMEP beamline of the Elettra Synchrotron (Trieste, Italy), all of whose members contributed to the success of these experiments, in particular Dr Diego Dreossi and Dr Nicola Sodini. Cell preparation and labelling, optimization of the sample preparation process and the in vivo animal procedures for the beamline experiments were performed at CBM and in the CBM Animal Facility, Trieste, Italy, situated in near proximity to the synchrotron. This unique situation was vital for this study, to ensure the usage of freshly prepared biological samples. The authors also gratefully acknowledge Dr Tim Gureyev (CSIRO, Australia) for fruitful discussions on the use of the phase-retrieval algorithms. In addition, the authors thank Stephen Kilfeather (Aeirtec; Newcastle, UK) for intense discussions and explanations of the pathomechanism of asthma.

\section{References}

Abràmoff, M. D., Magalhães, P. J. \& Ram, S. J. (2004). Biophoton. Int. 11, 36-42.

Ahrens, E. T. \& Bulte, J. W. M. (2013). Nat. Rev. Immunol. 13, 755763.

Al Heialy, S., Mcgovern, T. K. \& Martin, J. G. (2011). Respirology, 16, 589-597.

Arifin, D. R., Manek, S., Call, E., Arepally, A. \& Bulte, J. W. M. (2012). Biomaterials, 33, 4681-4689.

Astolfo, A., Schültke, E., Menk, R. H., Kirch, R. D., Juurlink, B. H., Hall, C., Harsan, L.-A., Stebel, M., Barbetta, D. \& Tromba, G. (2013). Nanomed. Nanotechnol. Biol. Med. 9, 284-292.

Bang, B. R., Chun, E., Shim, E. J., Lee, H. S., Lee, S. Y., Cho, S. H., Min, K. U., Kim, Y. Y. \& Park, H. W. (2011). Exp. Mol. Med. 43, 275-280.

Beltran, M. A., Paganin, D. M., Siu, K. K. W., Fouras, A., Hooper, S. B., Reser, D. H. \& Kitchen, M. J. (2011). Phys. Med. Biol. 56, 7353-7369.

Beltran, M. A., Paganin, D. M., Uesugi, K. \& Kitchen, M. J. (2010). Opt. Express, 18, 6423.
Biffi, S., Dal Monego, S., Dullin, C., Garrovo, C., Bosnjak, B., Licha, K., Welker, P., Epstein, M. M. \& Alves, F. (2013). PloS One, 8, e57150.

Blacquière, M. J., Hylkema, M. N., Postma, D. S., Geerlings, M., Timens, W. \& Melgert, B. N. (2010). Int. Arch. Allergy Immunol. 153, 173-181.

Bousso, P. \& Moreau, H. D. (2012). Nat. Rev. Immunol. 12, 858864.

Brun, F., Mancini, L., Kasae, P., Favretto, S., Dreossi, D. \& Tromba, G. (2010). Nucl. Instrum. Methods Phys. Res. A, 615, 326-332.

Chen, P. F., Luo, Y. L., Wang, W., Wang, J. X., Lai, W. Y., Hu, S. M., Cheng, K. F. \& Al-Abed, Y. (2010a). Mol. Med. 16, 400-408.

Chen, R.-C., Dreossi, D., Mancini, L., Menk, R., Rigon, L., Xiao, T.-Q. \& Longo, R. (2012). J. Synchrotron Rad. 19, 836-845.

Chen, R. C., Xie, H. L., Rigon, L., Longo, R., Castelli, E. \& Xiao, T. Q. (2011). Opt. Lett. 36, 1719-1721.

Chen, R., Xie, H., Rigon, L., Du, G., Castelli, E. \& Xiao, T. (2010b). Tsinghua Sci. Technol. 15, 102-107.

Cloetens, P., Ludwig, W., Baruchel, J., Van Dyck, D., Van Landuyt, J., Guigay, J. P. \& Schlenker, M. (1999b). Appl. Phys. Lett. 75, 2912.

Cloetens, P., Ludwig, W., Baruchel, J., Van Dyck, D., Van Landuyt, J., Guigay, J. P. \& Schlenker, M. (1999a). Proc. SPIE, 3772, 279-290.

Collins, T. J. (2007). BioTechniques, 43, 25-30.

Cormode, D. P., Naha, P. C. \& Fayad, Z. A. (2014). Contrast Media Mol. Imaging, 9, 37-52.

Cormode, D. P., Roessl, E., Thran, A., Skajaa, T., Gordon, R. E., Schlomka, J.-P., Fuster, V., Fisher, E. A., Mulder, W. J. M., Proksa, R. \& Fayad, Z. A. (2010). Radiology, 256, 774-782.

Cortez-Retamozo, V., Swirski, F. K., Waterman, P., Yuan, H., Figueiredo, J. L., Newton, A. P., Upadhyay, R., Vinegoni, C., Kohler, R., Blois, J., Smith, A., Nahrendorf, M., Josephson, L., Weissleder, R. \& Pittet, M. J. (2008). J. Clin. Invest. 118, 4058-4066.

Donnelly, E. F., Price, R. R. \& Pickens, D. R. (2003). Med. Phys. 30, 2888.

Dreossi, D., Abrami, A., Arfelli, F., Bregant, P., Casarin, K., Chenda, V., Cova, M. A., Longo, R., Menk, R., Quai, E., Quaia, E., Rigon, L., Rokvic, T., Sanabor, D., Tonutti, M., Tromba, G., Vascotto, A., Zanconati, F. \& Castelli, E. (2008). Eur. J. Radiol. 68, S58-S62.

Dunn, O. J. (1961). J. Am. Stat. Assoc. 56, 52-64.

Epstein, M. M. (2004). Int. Arch. Allergy Immunol. 133, 84-100.

Fullmer, H. M. (1960). J. Histochem. Cytochem. 8, 113-121.

Gelb, A. F., Licuanan, J., Shinar, C. M. \& Zamel, N. (2002). Chest, 121, 715-721.

Gelb, A. F. \& Zamel, N. (2002). Curr. Opin. Pulm. Med. 8, 50-53.

Golder, W., Viermetz, M. \& Gmeinwieser, J. (1991). Fortschr. Röntgenstr, 155, 562-567.

Gureyev, T. E., Mayo, S. C., Myers, D. E., Nesterets, Y., Paganin, D., Pogany, A., Stevenson, A. W. \& Wilkins, S. W. (2009). J. Appl. Phys. 105, 102005.

Gureyev, T., Mohammadi, S., Nesterets, Y., Dullin, C. \& Tromba, G. (2013). J. Appl. Phys. 114, 144906.

Heekeren, J. van, Kostenko, A., Hanashima, T., Yamada, H., Stallinga, S., Offerman, S. E. \& van Vliet, L. J. (2011). Med. Phys. 38, 5136.

Hildebrand, T. \& Ruegsegger, P. (1997). J. Microsc. 185, 67-75.

Hooper, S. B., Kitchen, M. J., Siew, M. L. L., Lewis, R. A., Fouras, A. B., te Pas, A., Siu, K. K. W., Yagi, N., Uesugi, K. \& Wallace, M. J. (2009). Clin. Exp. Pharmacol. Physiol. 36, 117-125.

Hooper, S. B., Kitchen, M. J., Wallace, M. J., Yagi, N., Uesugi, K., Morgan, M. J., Hall, C., Siu, K. K. W., Williams, I. M., Siew, M., Irvine, S. C., Pavlov, K. \& Lewis, R. A. (2007). FASEB J. 21, 33293337.

Keyriläinen, J., Bravin, A., Fernández, M., Tenhunen, M., Virkkunen, P. \& Suortti, P. (2010). Acta Radiol. 51, 866-884.

Kitchen, M. J., Lewis, R. A., Yagi, N., Uesugi, K., Paganin, D., Hooper, S. B., Adams, G., Jureczek, S., Singh, J., Christensen, C. R., Hufton, A. P., Hall, C. J., Cheung, K. C. \& Pavlov, K. M. (2005). Br. J. Radiol. 78, 1018-1027. 
Kitchen, M. J., Paganin, D., Lewis, R. A., Yagi, N., Uesugi, K. \& Mudie, S. T. (2004). Phys. Med. Biol. 49, 4335-4348.

Leong, K. P. \& Huston, D. P. (2001). Ann. Allergy Asthma Immunol. 87, 96-110.

Lewis, R. A., Yagi, N., Kitchen, M. J., Morgan, M. J., Paganin, D., Siu, K. K. W., Pavlov, K., Williams, I., Uesugi, K., Wallace, M. J., Hall, C. J., Whitley, J. \& Hooper, S. B. (2005). Phys. Med. Biol. 50, 50315040.

Markus, M. A., Dullin, C., Mitkovski, M., Prieschl-Grassauer, E., Epstein, M. M. \& Alves, F. (2014). PLoS ONE, 9, e90017.

Mbawuike, I. N. \& Herscowitz, H. B. (1989). J. Leukoc. Biol. 46, 119127.

Mizue, Y., Ghani, S., Leng, L., McDonald, C., Kong, P., Baugh, J., Lane, S. J., Craft, J., Nishihira, J., Donnelly, S. C., Zhu, Z. \& Bucala, R. (2005). Proc. Natl Acad. Sci. 102, 14410-14415.

Mohammadi, S., Larsson, E., Alves, F., Dal Monego, S., Biffi, S., Garrovo, C., Lorenzon, A., Tromba, G. \& Dullin, C. (2014). J. Synchrotron Rad. 21, 784-789.

Moreira, A. P. \& Hogaboam, C. M. (2011). J. Interferon Cytokine Res. 31, 485-491.

Mosmann, T. (1983). J. Immunol. Methods, 65, 55-63.

Nahrendorf, M., Zhang, H., Hembrador, S., Panizzi, P., Sosnovik, D. E., Aikawa, E., Libby, P., Swirski, F. K. \& Weissleder, R. (2008). Circulation, 117, 379-387.

Napp, J., Dullin, C., Müller, F., Uhland, K., Petri, J. B., van de Locht, A., Steinmetzer, T. \& Alves, F. (2010). Int. J. Cancer, 127, 19581974.

Nugent, K. A. (2007). J. Opt. Soc. Am. A, 24, 536-547.

Nugent, K. A. (2010). Adv. Phys. 59, 1-99.

Olendrowitz, C., Bartels, M., Krenkel, M., Beerlink, A., Mokso, R., Sprung, M. \& Salditt, T. (2012). Phys. Med. Biol. 57, 5309-5323.

Paganin, D., Mayo, S. C., Gureyev, T. E., Miller, P. R. \& Wilkins, S. W. (2002). J. Microsc. 206, 33-40,

Pfeiffer, F. Weitkamp, T. Bunk, O. \& David, C. (2006). Nat. Phys. 2 , 258-261.

Raupach, R. \& Flohr, T. G. (2011). Phys. Med. Biol. 56, 22192244.

Ryan, B. F., Joiner, B. L. \& Cryer, J. D. (2005). Minitab Handbook (updated for release 14). New York: Duxbury Press.
Schleede, S., Meinel, F. G., Bech, M., Herzen, J., Achterhold, K., Potdevin, G., Malecki, A., Adam-Neumair, S., Thieme, S. F., Bamberg, F., Nikolaou, K., Bohla, A., Yildirim, A. O., Loewen, R., Gifford, M., Ruth, R., Eickelberg, O., Reiser, M. \& Pfeiffer, F. (2012). Proc. Natl Acad. Sci. 109, 17880-17885.

Sera, T., Uesugi, K., Himeno, R. \& Yagi, N. (2007). Respir. Physiol. Neurobiol. 156, 304-311.

Sera, T., Uesugi, K. \& Yagi, N. (2005). Med. Phys. 32, 2787.

Takeda, T., Momose, A., Itai, Y., Wu, J. \& Hirano, K. (1995). Acad. Radiol. 2, 799-803.

Tanaka, T., Honda, C., Matsuo, S., Noma, K., Oohara, H., Nitta, N., Ota, S., Tsuchiya, K., Sakashita, Y., Yamada, A., Yamasaki, M., Furukawa, A., Takahashi, M. \& Murata, K. (2005). Invest. Radiol. 40, 385-396.

Taylor, L. (1981). IEEE Trans. Antennas Propag. 29, 386-391.

Thakur, M. L. (1977). Int. J. Appl. Radiat. Isot. 28, 183-201.

Tompkins, R. J., Mercer, I. P., Fettweis, M., Barnett, C. J., Klug, D. R., Porter, L. G., Clark, I., Jackson, S., Matousek, P., Parker, A. W. \& Towrie, M. (1998). Rev. Sci. Instrum. 69, 3113.

Trivedi, R. A., Mallawarachi, C., U-King-Im, J. M., Graves, M. J., Horsley, J., Goddard, M. J., Brown, A., Wang, L., Kirkpatrick, P. J., Brown, J. \& Gillard, J. H. (2006). Arterioscler. Thromb. Vasc. Biol. 26, 1601-1606.

Verdelis, K., Lukashova, L., Atti, E., Mayer-Kuckuk, P., Peterson, M. G. E., Tetradis, S., Boskey, A. L. \& van der Meulen, M. C. H. (2011). Bone, 49, 580-587.

Washko, G. R., Parraga, G. \& Coxson, H. O. (2012). Respirology, 17, 432-444.

Weitkamp, T., Raven, C. \& Snigirev, A. A. (1999). Proc. SPIE, 3772, 311-317.

Wu, X., Liu, H. \& Yan, A. (2005). Opt. Lett. 30, 379-381.

Yagi, N., Suzuki, Y., Umetani, K., Kohmura, Y. \& Yamasaki, K. (1999). Med. Phys. 26, 2190.

Yang, M., Kumar, R. K., Hansbro, P. M. \& Foster, P. S. (2012). J. Leukoc. Biol. 91, 557-569.

Yong, H. S., Kang, E. Y., Kim, Y. K., Woo, O. H., Shin, B. K., Oh, C. H., Je, J. H., Han, H. \& Seo, J. S. (2009). Yonsei Med. J. 30, 422 426.

Zhang, L., Li, D. \& Luo, S. (2011). PLoS ONE, 6, e17400. 